\title{
Endovascular thrombectomy for M2 occlusions: comparison between forced arterial suction thrombectomy and stent retriever thrombectomy
}

\author{
Yong-Won Kim, ${ }^{1,2}$ Seungnam Son, ${ }^{3}$ Dong-Hun Kang, ${ }^{2,4}$ Yang-Ha Hwang, ${ }^{1}$ \\ Yong-Sun $\mathrm{Kim}^{2}$
}

'Department of Neurology, Kyungpook National University Hospital, Daegu, Republic of Korea

2Department of Radiology, Kyungpook National University Hospital, Daegu, Republic of Korea

${ }^{3}$ Department of Neurology, Jinju Hanil Hospital, Jinju, Republic of Korea

${ }^{4}$ Department of Neurosurgery, Kyungpook National University Hospital, Daegu, Republic of Korea

Correspondence to Professor Yang-Ha Hwang, Department of Neurology, Kyungpook National University Hospital, School of Medicine, Kyungpook National University, Daegu, Republic of Korea, 130, Dongduk-ro, Jung-gu, Daegu 41944, Republic of Korea; yangha.hwang@gmail.com

Received 14 April 2016 Revised 2 June 2016 Accepted 13 June 2016 Published Online First 5 July 2016

\section{ABSTRACT}

Background To date there has been no direct comparison of two frequently used endovascular thrombectomy (EVT) methods (forced arterial suction thrombectomy (FAST) and stent retriever thrombectomy) in M2 occlusions. We review our experiences with EVT performed using FAST and stent retriever thrombectomy in such cases.

Methods The subjects comprised 41 patients with an M2 occlusion who underwent EVT (25 with FAST, 16 with stent retriever thrombectomy). The patients' data were retrospectively analyzed to evaluate the technical characteristics and angiographic outcome of the two EVT techniques.

Results Thrombolysis In Cerebral Infarction (TICI) grades $2 b-3$ using the first chosen technique did not differ significantly between the two techniques (FAST $64.0 \%$ vs stent retriever thrombectomy $81.2 \%$, $p=0.305$ ). Time from groin puncture to reperfusion was significantly shorter for stent retriever thrombectomy (53.0 vs 38.5 min; $p=0.045$ ). Distal embolization occurred in three cases $(12.0 \%)$ in the FAST group and in four $(26.7 \%)$ in the stent retriever group ( $p=0.362$ ). However, the two techniques did not differ significantly in the final $\mathrm{TICl} 2 \mathrm{~b}-3$ rate $(72.0 \%$ vs $87.5 \% ; \mathrm{p}=0.441)$. A frequent angiographic finding regarding the failure of FAST was that the M2 occlusion was located immediately after severe acute angulation between M1 and $\mathrm{M} 2$.

Conclusions Stent retriever thrombectomy may provide faster reperfusion than FAST, while the FAST technique might be associated with lower distal embolization and a higher reperfusion rate for the first thrombectomy attempt, but without any significant difference in clinical outcome. When choosing the EVT method for M2 occlusions, consideration of the location of the occlusion and tortuosity between M1 and M2 might be helpful to achieve a better angiographic outcome.

\section{INTRODUCTION}

Recent trials regarding endovascular thrombectomy (EVT) in patients with acute ischemic stroke (AIS) due to proximal artery occlusion in the anterior circulation have demonstrated its benefits in terms of efficacy and safety when used with or without intravenous thrombolytic therapy. ${ }^{1-5}$ Based on data from these trials, updated guidelines on EVT for patients with AIS recommend using the stent retriever for occlusions of a proximal intracranial artery such as the distal internal carotid artery
(ICA) and proximal middle cerebral artery (MCA). ${ }^{67}$

However, the risks and benefits of EVT for distal intracranial arterial occlusions (ie, M2 or M3 segments of the MCA) still remain debatable. Theoretically, the size of the ischemic core and ischemic penumbra for a distal intracranial occlusion is less than that of a proximal intracranial arterial occlusion; this difference can lessen the beneficial effects of EVT for M2 occlusions, while the thin vessel wall and narrow lumen of distal intracranial arteries can be associated with a higher risk of EVT complications.

Despite the lack of clarity regarding its benefits, the use of EVT with stent retrievers is recommended by the updated American Heart Association/ American Stroke Association Korean Clinical Practice guidelines in carefully selected patients with AIS due to $\mathrm{M} 2$ segment occlusion. ${ }^{67} \mathrm{~A}$ few studies have retrospectively investigated the safety and efficacy of EVT in patients with M2 occlusions. ${ }^{8-11}$ These studies have examined the clinical outcome following EVT of $\mathrm{M} 2$ occlusions and have mostly used stent retriever thrombectomy as the EVT method.

To date there has been no direct comparison of two frequently used EVT methods (stent retriever and aspiration thrombectomy using a large-bore catheter) in patients with $\mathrm{M} 2$ occlusions. We therefore retrospectively analyzed the data of 41 patients with AIS due to occlusion of the M2 segment of the MCA who underwent EVT using a stent retriever or forced arterial suction thrombectomy (FAST) with the Penumbra reperfusion catheter. We compared the safety and efficacy of the two EVT methods in terms of technical characteristics. In addition, we aimed to identify predictors of a favorable clinical outcome after EVT in M2 occlusions.

\section{MATERIALS AND METHODS Patients}

The subjects for this study were chosen from patients with acute stroke treated at our institute between January 2011 and June 2015 as per the prospectively recorded acute stroke endovascular treatment registry maintained at our center. The criteria for inclusion in the study were as follows: (1) Patients had AIS with angiographically confirmed M2 segment occlusion and National Institute of Health Stroke Scale (NIHSS) score of $\geq 6$ at the time of arrival. The M2 segment was defined as part of the MCA extending from the 
genu to the top of the Sylvian fissure and the circular sulcus. ${ }^{12}$ (2) Time from symptom onset to hospital arrival was $<8$ hours. (3) EVT was performed using the FAST technique with the Penumbra reperfusion catheter or the stent retriever technique. The study protocol and study design of retrospective analysis was approved by the local institutional board.

\section{Acute stroke management and endovascular procedure}

The initial neurological examination, including the assessment of the NIHSS score, was performed in the emergency room. Non-enhanced brain CT scans were performed immediately to rule out intracerebral hemorrhage (ICH). If no ICH was evident on CT scans, intravenous recombinant tissue plasminogen activator (rtPA; Actilyse; Boehringer Ingelheim, Basel, Switzerland) was administered in accordance with the guidelines for early management (within 3 hours of symptom onset up to December 2012 and within 4.5 hours since January 2013 ) of adults with ischemic stroke. ${ }^{13}$ Vascular imaging (CT angiography or MR angiography) and diffusion-weighted MRI were conducted subsequently. The institutional criteria for EVT were as follows: (1) AIS corresponding to arterial occlusion on CT angiography or MR angiography; (2) infarction volume on CT or diffusion-weighted MRI less than half of the corresponding vascular territory; and (3) no evidence of welldeveloped parenchymal hyperintensity on FLAIR or T2-weighted imaging in patients $>6$ hours from symptom onset.

Informed consent was obtained before initiating EVT. All interventional procedures were performed by three experienced neurointerventionalists (Y-WK, D-HK, and Y-SK) using a biplane angiography system. Transfemoral cerebral angiography was performed under conscious sedation. EVT was initiated after the assessment of the angioarchitecture of the occluded artery and collateral status. A 9 Fr balloon-tipped guide catheter (BGC; Optimo, Tokai Medical, Aichi, Japan) was advanced to the most distal portion of the accessible intracranial ICA.

The choice of EVT technique in each case was made according to the neurointerventionists' preferences, except that the stent retriever was used in cases when the diameter of M2 was smaller than the Penumbra reperfusion catheter.

For the FAST technique, the Penumbra reperfusion catheter (041 or 4Max; Penumbra, Alameda, California, USA) coaxially with a 0.014 inch microguidewire (Synchro-14; Stryker, Kalamazoo, Michigan, USA or Traxcess 14; Microvention, Columbia, USA) and a 0.021 inch microcatheter (Prowler Select Plus; Cordis, Miami, Florida, USA) were advanced to the proximal end of the occlusion and wedged to the clot under manual aspiration using a $50 \mathrm{~mL}$ syringe. After the balloon of the BGC was inflated, the Penumbra reperfusion catheter was withdrawn while continuing to maintain manual aspiration. ${ }^{14}$

For the stent retriever technique (Solitaire AB/FR; Covidien-Medtronic, Irvine, California, USA or TrevoProVue/ XP; Stryker, Kalamazoo, Michigan, USA), the target occluded segment was navigated with a 0.014 inch microguidewire and a 0.021 inch microcatheter was advanced beyond the occlusion. The proximal and distal ends of the occlusion were then confirmed, and the stent retriever was deployed for several minutes. After the balloon of the BGC was inflated with remote aspiration through the guide catheter, the microcatheter and stent retriever were withdrawn into the guide catheter. If reperfusion was not achieved after three attempts of mechanical thrombectomy, we used rescue measures such as switching to another mechanical thrombectomy device ${ }^{15}$ and intra-arterial tirofiban infusion. ${ }^{16}$

\section{Clinical and angiographic data collection}

Data for the following parameters were collected from the patient records: demographics, clinical characteristics, complete medical history, and angiography findings. The NIHSS score was assessed at baseline and at day 7. The Alberta Stroke Program Early CT Score (ASPECTS) was calculated from initial diffusion-weighted image (DWI). The clinical outcome was assessed using the modified Rankin Scale (mRS) at 3 months, and a favorable clinical outcome was defined as a mRS score of $\leq 2$. The reperfusion status was assessed using the Thrombolysis In Cerebral Infarction (TICI) score, which was determined during the final angiography after thrombectomy. The TICI grade was independently evaluated by an experienced neuroradiologist (Y-SK) and neurologist (Y-HH) who were blinded to the patients' clinical information. If there was a discordance, the final assessment was determined by consensus. Successful reperfusion was defined as a TICI score of $2 b$ or 3 .

A control CT scan was acquired immediately after the EVT and 24 hours after the procedure to check for hemorrhagic complications. The type of intracranial hemorrhage was determined according to the European Cooperative Acute Stroke Study II classification system. ${ }^{17}$ Symptomatic intracranial hemorrhage was defined as any type of hemorrhage with an increase of $\geq 4$ in NIHSS score within 24 hours. ${ }^{18}$

\section{Statistical analysis}

Statistical analyses were performed using SPSS V.22.0 (IBM, Armonk, New York, USA). A $\chi^{2}$ test or Fisher exact test was used for categorical data. The Student t-test or Mann-Whitney $\mathrm{U}$ test was used for continuous variables. Multiple linear regression analysis was performed to identify any independent predictor(s) of favorable clinical outcome at 3 months. Age, DWI-ASPECTS, initial NIHSS score, use of intravenous rtPA, EVT device, time from symptom onset to reperfusion, and reperfusion of TICI $2 b-3$ were included as potential confounders. A $p$ value of $<0.05$ was considered statistically significant.

\section{RESULTS}

\section{Overall results}

The median age of the 41 enrolled patients was 72 years (IQR 64-79) and the median NIHSS score was 13 (IQR 8-17). Twenty-six patients had left MCA M2 occlusions and 20 patients $(48.8 \%)$ received intravenous rtPA prior to EVT. The median time from symptom onset to door was $142 \mathrm{~min}$ and the median time from door to groin puncture was 99 min. Baseline characteristics are summarized in table 1.

The first attempt at EVT was made with the Penumbra reperfusion catheter via the FAST technique in 25 patients $(61.0 \%)$ and with the stent retriever technique in the remaining 16 patients (39.0\%). For 70.7\% (29/41) of the patients, successful reperfusion (TICI 2b-3) was achieved using the first choice device (primary reperfusion), while $17.1 \%$ (7/41) of the patients required rescue measures. The median time from groin puncture to reperfusion was $45 \mathrm{~min}$. The successful reperfusion rate, as per the final angiography, was $78.0 \%$ and one patient $(2.4 \%)$ developed symptomatic ICH. The median NIHSS score at 7 days after stroke onset was 2 (IQR 1-6), and a favorable clinical outcome at 3 months was achieved in 33 patients (80.5\%).

\section{Technical comparison between the FAST and stent retriever techniques}

No statistically significant differences were noted between the FAST technique and the stent retriever technique in terms of 
Ischemic stroke

Table 1 Baseline characteristics

\begin{tabular}{|c|c|c|c|}
\hline Variable & FAST & Stent retriever & p Value \\
\hline Number & 25 & 16 & \\
\hline Age, median (IQR) & $71(60.5-75)$ & $76.5(65.5-82)$ & $0.121^{*}$ \\
\hline Female & $8(32 \%)$ & $9(56.2 \%)$ & 0.124 \\
\hline NIHSS, median (IQR) & $15(8-17)$ & $10.5(6-17)$ & $0.511^{*}$ \\
\hline $\begin{array}{l}\text { NIHSS at } 7 \text { days, median } \\
\text { (IQR) }\end{array}$ & $2(1-6)$ & $1.5(0.25-5.75)$ & $0.465^{*}$ \\
\hline DWI-ASPECTS score & $8(7.5-9)$ & $7(6.75-9.0)$ & $0.230^{*}$ \\
\hline Lesion side (left) & $14(56 \%)$ & $12(75 \%)$ & 0.218 \\
\hline Intravenous tPA & $13(52 \%)$ & $7(43.8 \%)$ & 0.606 \\
\hline Hypertension & $15(60 \%)$ & $11(68.8 \%)$ & 0.570 \\
\hline Diabetes & $5(20 \%)$ & $3(18.8 \%)$ & $>0.999 \dagger$ \\
\hline Dyslipidemia & $6(24 \%)$ & $7(43.8 \%)$ & 0.185 \\
\hline Atrial fibrillation & $15(60 \%)$ & $12(75 \%)$ & 0.323 \\
\hline Coronary disease & $2(8 \%)$ & $2(12.5 \%)$ & $0.637 \dagger$ \\
\hline Previous stroke & $3(12.0 \%)$ & $2(12.5 \%)$ & $>0.999 \dagger$ \\
\hline Smoking & $7(28.0 \%)$ & $3(18.8 \%)$ & $0.712 \dagger$ \\
\hline $\begin{array}{l}\text { Onset to door time, median } \\
\text { (IQR) }\end{array}$ & $137(47-307)$ & $143.5(77.5-299.25)$ & $0.897^{*}$ \\
\hline
\end{tabular}

*Mann-Whitney U test.

tFisher's exact t-test.

ASPECTS, Alberta Stroke Program Early CT Score; DWI, diffusion-weighted image;

FAST, forced arterial suction thrombectomy; NIHSS, National Institute of Health Stroke

Scale; tPA, tissue plasminogen activator. the baseline characteristics. EVT device delivery time from guide catheter to target occlusion did not differ significantly between the two methods ( $4 \mathrm{~min}$ for FAST vs $3 \mathrm{~min}$ for the stent retriever, $\mathrm{p}=0.120$ ). Both EVT techniques had a similar rate of successful reperfusion at the first thrombectomy attempt $(56.0 \%$ vs $56.2 \%, \mathrm{p}=0.987)$ and with the primary chosen method $(64.0 \%$ vs $81.2 \%, \mathrm{p}=0.305)$. The rate of successful reperfusion in less than $30 \mathrm{~min}$ from carotid catheterization to reperfusion was not significantly different $(64.0 \%$ vs $75.0 \%$, $\mathrm{p}=0.460)$. Five $(19.2 \%)$ of the 26 patients in the FAST group required rescue treatment, including device switching to the stent retriever with intra-arterial tirofiban infusion, and two patients $(12.5 \%)$ required conversion from the stent retriever technique to the FAST technique. The time from groin puncture to reperfusion was significantly shorter using the stent retriever technique (median 53 vs $38.5 \mathrm{~min}$; $\mathrm{p}=0.045$ ). However, the two techniques did not show a statistically significant difference in the final reperfusion rate $(72.0 \%$ vs $87.5 \% ; \mathrm{p}=0.441)$ and a favorable clinical outcome at 3 months $(84.0 \%$ vs $75.0 \%$; $\mathrm{p}=0.689$ ). There was no embolization of uninvolved territory in either group, but distal embolization occurred in three cases $(12.0 \%)$ in the FAST group and in four cases $(26.7 \%)$ in the stent retriever group $(p=0.362)$. Symptomatic ICH occurred in one patient $(4.0 \%)$ treated with the FAST technique. Angiographic data and clinical outcomes are summarized in table 2. Multiple linear regression analysis showed that age

Table 2 Details of endovascular treatment and clinical outcomes

\begin{tabular}{|c|c|c|c|}
\hline Variable & FAST & Stent retriever & p Value \\
\hline Onset to door time, median (IQR) & $137(47-307)$ & $143.5(77.5-299.25)$ & $0.897^{*}$ \\
\hline Door to puncture time, median (IQR) & $99(82.5-120.5)$ & $98(66.5-123.25)$ & $0.626^{*}$ \\
\hline Puncture to recanalization time, median (IQR) & $53(41-74)$ & $38.5(31.5-57.25)$ & $0.045^{*}$ \\
\hline \multicolumn{4}{|l|}{ Time intervals, median (IQR) } \\
\hline Puncture to carotid catheterization & $24(16-35)$ & $20.5(15.25-23)$ & $0.080^{*}$ \\
\hline Guide catheter to access to the target occlusion & $4(2.5-7.5)$ & $3(2-4)$ & $0.120^{*}$ \\
\hline Start of thrombectomy to recanalization & $16(7-42.5)$ & $15.5(12.25-33.5)$ & $0.678^{*}$ \\
\hline $\mathrm{TICl} 2 \mathrm{~b}-3$ by first thrombectomy attempt & $14(56.0 \%)$ & $9(56.2 \%)$ & 0.987 \\
\hline $\mathrm{TICl} 2 \mathrm{~b}-3$ by primary chosen device & $16(64.0 \%)$ & $13(81.2 \%)$ & $0.305 t$ \\
\hline $\mathrm{TICl} 2 \mathrm{~b}-3<60$ min (puncture to recanalization) & $15(60.0 \%)$ & $12(75.0 \%)$ & 0.323 \\
\hline $\mathrm{TICl} 2 \mathrm{~b}-3<30 \mathrm{~min}$ (carotid catheterization to recanalization) & $16(64.0 \%)$ & $12(75.0 \%)$ & 0.460 \\
\hline Rescue treatments used & $5(20.0 \%)$ & $2(12.5 \%)$ & $0.685 t$ \\
\hline Stent retriever & 5 & 0 & \\
\hline FAST & 0 & 2 & \\
\hline Intra-arterial tirofiban & 5 & 0 & \\
\hline Final $\mathrm{TICl} 2 \mathrm{~b}-3$ & $18(72.0 \%)$ & $14(87.5 \%)$ & $0.441 \dagger$ \\
\hline Distal embolization & $3(12.0 \%)$ & $4(26.7 \%)$ & $0.392 \dagger$ \\
\hline Uninvolved territory embolization & 0 & 0 & \\
\hline \multicolumn{4}{|l|}{ Intracranial hemorrhage } \\
\hline Hemorrhagic infarction 1 & $3(12.0 \%)$ & $2(12.5 \%)$ & $1.000 t$ \\
\hline Hemorrhagic infarction 2 & 0 & $1(6.2 \%)$ & $0.390 \dagger$ \\
\hline Parenchymal hematoma 1 & 0 & 0 & \\
\hline Parenchymal hematoma 2 & $1(4.0 \%)$ & 0 & $1.000 t$ \\
\hline Symptomatic ICH & $1(4.0 \%)$ & 0 & $1.000 t$ \\
\hline SAH & 0 & $1(6.2 \%)$ & $0.390 t$ \\
\hline mRS $0-2$ at 3 months & $21(84.0 \%)$ & $12(75.0 \%)$ & $0.689 \dagger$ \\
\hline Mortality & 0 & $1(6.2 \%)$ & $0.390 t$ \\
\hline
\end{tabular}

*Mann-Whitney U test.

tFisher's exact t-test.

FAST, forced arterial suction thrombectomy; ICH, intracerebral hemorrhage; mRS, modified Rankin Scale; SAH, subarachnoid hemorrhage; TICl, Thrombolysis in Cerebral Infarction. 
Table 3 Multiple linear regression analysis for favorable clinical outcome at 3 months

\begin{tabular}{|c|c|c|c|c|c|c|}
\hline \multirow[b]{2}{*}{ Variables } & \multicolumn{2}{|c|}{$\begin{array}{l}\text { Unstandardized } \\
\text { coefficients }\end{array}$} & \multirow{2}{*}{$\begin{array}{l}\text { Standardized } \\
\text { coefficients }\end{array}$} & \multirow[b]{2}{*}{$p$ Value } & \multicolumn{2}{|l|}{$95 \% \mathrm{Cl}$} \\
\hline & B & S.E & & & Lower & Upper \\
\hline Age (/1 year) & -0.016 & 0.006 & -0.463 & 0.009 & -0.028 & -0.004 \\
\hline DWI-ASPECTS & 0.062 & 0.046 & 0.233 & 0.187 & -0.032 & 0.157 \\
\hline Initial NIHSS & -0.002 & 0.012 & -0.028 & 0.875 & -0.027 & 0.023 \\
\hline Intravenous tPA & -0.083 & 0.145 & -0.105 & 0.572 & -0.380 & 0.214 \\
\hline EVT strategy & -0.043 & 0.148 & -0.050 & 0.772 & -0.346 & 0.260 \\
\hline $\begin{array}{l}\text { Onset to } \\
\text { reperfusion } \\
\text { time(/10 min) }\end{array}$ & -0.005 & 0.002 & -0.414 & 0.027 & -0.010 & -0.001 \\
\hline $\begin{array}{l}\text { Successful } \\
\text { reperfusion }\end{array}$ & 0.092 & 0.143 & 0.096 & 0.528 & -0.202 & 0.385 \\
\hline
\end{tabular}

ASPECTS, Alberta Stroke Program Early CT Score; DWI, diffusion-weighted image; EVT, endovascular thrombectomy; NIHSS, National Institute of Health Stroke Scale; tPA, tissue plasminogen activator.

$(\beta=-0.463, p=0.009)$ and time from symptom onset to reperfusion $(\beta=-0.414, p=0.027)$ were associated with a favorable clinical outcome at 3 months (table 3 ).

\section{DISCUSSION}

In this study the rates of successful reperfusion and favorable outcome following EVT for patients with an M2 occlusion were $78.6 \%$ and $81.0 \%$, respectively, with a mortality rate of $2.4 \%$. These results are comparable with those reported in recent retrospective studies on EVT performed using only the stent retriever technique for M2 segment occlusions. ${ }^{8-10}$ In this study we did not find any significant difference between the two evaluated techniques in terms of reperfusion rate or rate of favorable clinical outcome. However, time from groin puncture to reperfusion was significantly shorter using the stent retriever technique than with FAST. The FAST technique had a lower rate of distal embolization than the stent retriever technique, although the difference was not statistically significant.

Our study design did not allow for the assessment of the superiority of either EVT technique because there was no control group receiving medical treatment alone or treatment using other EVT techniques. To overcome this deficiency, we reviewed historical data involving various EVT strategies to treat M2 occlusions. One study on the natural history of acute stroke indicated a poor clinical outcome (mRS score $\geq 3$ ) at 3 months in up to $47 \%$ of patients with a distal MCA occlusion not administered reperfusion therapy. ${ }^{19}$ Furthermore, studies on patients with distal MCA occlusions receiving intravenous thrombolytic agents found reperfusion rates of $30.8-68.4 \%$ and favorable clinical outcomes ranging from $48 \%$ to $81 \% .^{20-22}$ Finally, a recent study on EVT using the stent retriever technique reported reperfusion rates of $78.5-93.3 \%$ and a favorable clinical outcome in 60-66.3\% of M2 occlusion cases (table 4). ${ }^{8-10}$ These results are comparable with the overall result of the present study, which yielded a final successful reperfusion rate of $78.6 \%$ and a favorable clinical outcome in $81.0 \%$.

For comparison of the technical characteristics of the two EVT techniques, we analyzed key time intervals of EVT in terms of puncture to carotid catheterization time, guide catheter to target access time, and target access to reperfusion time. With regard to the delivery of the thrombectomy device, it is known that the Penumbra reperfusion catheter is more difficult than the stent retriever because of its larger size compared with the microcatheter in stent retriever thrombectomy. ${ }^{23}$ The tortuosity of the carotid siphon may impede the delivery of the Penumbra catheter to the occlusion site using the FAST technique, as was the case with the origin of the ophthalmic artery. However, our results showed no significant difference in time from guide catheter to target access. The Penumbra reperfusion catheter itself has a highly navigable flexible tip, and it could help to overcome such impedance with the use of a coaxial system $(0.021$ inch microcatheter and 0.014 inch microwire) and steam shaping of the catheter tip. In terms of the reperfusion process, time from the start of thrombectomy to final reperfusion was similar for both EVT techniques. Among the cases with successful reperfusion, the FAST technique achieved a higher reperfusion rate with first thrombectomy attempts than the stent retriever $(77.8 \%$ vs $64.3 \%)$, although it had a lower final successful reperfusion rate. This finding suggests that the FAST technique might be less effective for repeated thrombectomy attempts. Therefore, early switching to other techniques might provide better angiographic outcomes in cases with unsuccessful reperfusion after a few attempts with the FAST technique.

Based on the review of angiographic data, we found that successful or unsuccessful reperfusion using the FAST technique was associated with the location of the thrombus and the tortuosity between M1 and M2. If the thrombus was located immediately after severe acute angulation, especially interposition from M1 to M2, it would disturb the optimal contact of the Penumbra catheter tip and the thrombus. Eventually, the FAST technique might be ineffective due to imprecise facing with the thrombus and Penumbra catheter tip. In these cases, we assume that it might be reasonable to use the stent retriever instead of the Penumbra reperfusion catheter.

We used a balloon guide catheter in both groups. There was no embolization of uninvolved territory in either group, but distal embolization occurred in $12 \%$ of cases in the FAST group and in $26.7 \%$ of cases in the stent retriever group. For our FAST technique, we did not cross the clot using a microcatheter and microguidewire so fragmentation or disruption of the clot may rarely occur (figure 1). We also believe that the small clot size in an M2 occlusion, which could be easily removed by

Table 4 Characteristics of reperfusion therapy studies for M2 occlusions

\begin{tabular}{|c|c|c|c|c|c|c|}
\hline Studies & Age & Female (\%) & Baseline NIHSS & Reperfusion method & Reperfusion status & $\mathrm{mRS} \leq 2$ at 3 months $(\%)$ \\
\hline Flores et $a l^{10}(n=65)$ & 66 (mean) & 47.7 & 16 (median) & Stent retriever \pm IA-tPA $\pm I V-t P A$ & $78.5 \%(\mathrm{TICl} 2 \mathrm{~b}-3)$ & 60 \\
\hline Dorn et $a l^{9}(n=16)$ & 68.1 (mean) & N/A & 13.7 (mean) & Stent retriever \pm IV-tPA & $93.3 \%(\mathrm{TICl} 2 \mathrm{~b}-3)$ & 60 \\
\hline Coutinho et $a l^{8}(n=50)$ & 71 (mean) & 48 & 13 (median) & MERCI, Solitaire \pm IV-tPA & $85 \%(\mathrm{mTICl} 2 \mathrm{~b}-3)$ & 60 \\
\hline Current study ( $n=41)$ & 72 (median) & 41.5 & 13 (median) & FAST, Stent retriever $\pm I V-t P A$ & $78 \%(\mathrm{TICl} 2 \mathrm{~b}-3)$ & 80.5 \\
\hline
\end{tabular}

FAST, forced arterial suction thrombectomy; IA, intra-arterial; IV, intravenous; mRS; modified Rankin Scale; mTICI, modified thrombolysis in cerebral ischemia; NIHSS, National Institute of Health Stroke Scale; tPA, tissue plasminogen activator; TICI, Thrombolysis In Cerebral Infarction. 
suction power via the Penumbra catheter, could also be a reason for less distal embolization in the FAST group.

In this study we noted a discrepancy in the results regarding the reperfusion rate and rate of favorable outcome between the two EVT techniques. Generally, a higher reperfusion rate is associated with better clinical outcomes. ${ }^{1-5}$ However, the rate of favorable clinical outcome was lower for the stent retriever technique than the FAST technique, despite a higher reperfusion rate. Various factors including arterial occlusion site, infarct volume, collateral status, use of rtPA, reperfusion status, time from symptom onset to reperfusion, and age have been established as predictors of clinical outcome in other studies. $^{21}{ }^{24-27}$ In this study, multivariate analysis showed that age and time from symptom onset to reperfusion were the independent predictors of favorable outcomes in EVT for M2 segment occlusion. Additionally, the absolute value of the $\beta$ coefficient of age was greater than that of the time from symptom onset to reperfusion. We therefore speculate that age had the greatest impact on favorable clinical outcomes in our study, so the worse clinical outcomes in the stent retriever group-despite better reperfusion rates-can be explained by the fact that the patients in the stent retriever group had an older median age.

This study has some limitations. First, this was a retrospective single-center study although the data were collected prospectively, so there is a possibility of selection bias. Second, the sample size was small so the statistical power of the study might be weak. Third, there was no control group that did not undergo EVT, so we were not in a position to assess whether EVT should be done using FAST or the stent retriever technique.

\section{CONCLUSIONS}

Our findings suggest that EVT for M2 segment occlusion may be safe and feasible. EVT using stent retrievers was associated with more rapid reperfusion, while the FAST technique had a higher reperfusion rate for first thrombectomy attempts. There was no significant difference in terms of the clinical outcome between the two groups. In EVT for M2 occlusions, choosing the EVT technique based on the location of occlusion and the tortuosity between the M1 and M2 segments might result in better angiographic outcomes. Future prospective multicenter studies are necessary to further confirm our findings.

Twitter Follow Yang-Ha Hwang at @yangha73

Acknowledgements The authors thank Wade Martin of Emareye Medical Editing for his critical English revision.

Contributors Study concept and design: Y-WK, Y-HH; Data collection: Y-WK, D-HK, Y-HH; Drafting of the manuscript: Y-WK, SS; Critical revision of the manuscript for important intellectual content: Y-HH, Y-SK.

Competing interests None declared.

Provenance and peer review Not commissioned; externally peer reviewed.

\section{REFERENCES}

1 Berkhemer OA, Fransen PSS, Beumer D, et al. A randomized trial of intraarterial treatment for acute ischemic stroke. N Engl J Med 2015;372:11-20.

2 Goyal M, Demchuk AM, Menon BK, et al. Randomized assessment of rapid endovascular treatment of ischemic stroke. N Engl J Med 2015;372:1019-30.
3 Saver JL, Goyal M, Bonafé A, et al. Stent-retriever thrombectomy after intravenous t-PA vs. t-PA alone in stroke. N Engl J Med 2015;372:2285-95.

4 Jovin TG, Chamorro A, Cobo $E$, et al. Thrombectomy within 8 hours after symptom onset in ischemic stroke. N Engl J Med 2015;372:2296-306.

5 Campbell BCV, Mitchell PJ, Kleinig TJ, et al. Endovascular therapy for ischemic stroke with perfusion-imaging selection. N Engl J Med 2015;372:1009-18.

6 Powers WJ, Derdeyn CP, Biller J, et al. 2015 American Heart Association/American Stroke Association focused update of the 2013 guidelines for the early management of patients with acute ischemic stroke regarding endovascular treatment. Stroke 2015;46:3020-35.

7 Hong KS, Ko SB, Yu KH, et al. Update of the Korean clinical practice guidelines for endovascular recanalization therapy in patients with acute ischemic stroke. J Stroke 2016;18:102-13.

8 Coutinho JM, Liebeskind DS, Slater LA, et al. Mechanical thrombectomy for Isolated M2 occlusions: a post hoc analysis of the STAR, SWIFT, and SWIFT PRIME studies. AJNR Am J Neuroradiol 2016;37:667-72.

9 Dorn $\mathrm{F}$, Lockau $\mathrm{H}$, Stetefeld $\mathrm{H}$, et al. Mechanical thrombectomy of M2-occlusion. J Stroke Cerebrovasc Dis 2015;24:1465-70.

10 Flores A, Tomasello A, Cardona P, et al. Endovascular treatment for M2 occlusions in the era of stentrievers: a descriptive multicenter experience. J Neurointerv Surg 2015:7:234-7.

11 Munich SA, Hall SL, Cress MC, et al. To treat or not to treat M2 occlusions? The question (and answer) from a single institution. Neurosurgery Published Online First: 14 December 2015.

12 Osborn AG. Diagnostic cerebral angiography. 2nd edn. Philadelphia, PA: Lippincott Williams \& Wilkins, 1999:135-8.

13 Kernan WN, Ovbiagele B, Black HR, et al. Guidelines for the prevention of stroke in patients with stroke and transient ischemic attack: a guideline for healthcare professionals from the American Heart Association/American Stroke Association. Stroke 2014;45:2160-236.

14 Kang DH, Hwang YH, Kim YS, et al. Direct thrombus retrieval using the reperfusion catheter of the penumbra system: forced-suction thrombectomy in acute ischemic stroke. AJNR Am J Neuroradiol 2011;32:283-7.

15 Kang DH, Kim YW, Hwang YH, et al. Switching strategy for mechanical thrombectomy of acute large vessel occlusion in the anterior circulation. Stroke 2013;44:3577-9.

16 Kang DH, Kim YW, Hwang YH, et al. Instant reocclusion following mechanical thrombectomy of in situ thromboocclusion and the role of low-dose intra-arterial tirofiban. Cerebrovasc Dis 2014;37:350-5.

17 Hacke W, Kaste M, Fieschi C, et al. Randomised double-blind placebo-controlled trial of thrombolytic therapy with intravenous alteplase in acute ischaemic stroke (ECASS II). Second European-Australasian Acute Stroke Study Investigators. Lancet 1998;352:1245-51.

18 Saver JL, Jahan R, Levy El, et al. Solitaire flow restoration device versus the Merci Retriever in patients with acute ischaemic stroke (SWIFT): a randomised, parallel-group, non-inferiority trial. Lancet 2012;380:1241-9.

19 Hernandez-Pérez M, Pérez de la Ossa N, Aleu A, et al. Natural history of acute stroke due to occlusion of the middle cerebral artery and intracranial internal carotid artery. J Neuroimaging 2014;24:354-8.

20 Bhatia R, Hill MD, Shobha N, et al. Low Rates of acute recanalization with intravenous recombinant tissue plasminogen activator in ischemic stroke: real-world experience and a call for action. Stroke 2010;41:2254-8.

21 Saqqur M, Uchino K, Demchuk AM, et al. Site of arterial occlusion identified by transcranial Doppler predicts the response to intravenous thrombolysis for stroke. Stroke 2007;38:948-54.

22 Zangerle $A$, Kiechl S, Spiegel $M$, et al. Recanalization after thrombolysis in stroke patients: predictors and prognostic implications. Neurology 2007;68:39-44.

23 Spiotta AM, Chaudry MI, Hui FK, et al. Evolution of thrombectomy approaches and devices for acute stroke: a technical review. J Neurointerv Surg 2015;7:2-7.

24 Demchuk AM, Goyal M, Yeatts SD, et al. Recanalization and clinical outcome of occlusion sites at baseline CT angiography in the Interventional Management of Stroke III trial. Radiology 2014;273:202-10.

25 Mazighi M, Chaudhry SA, Ribo M, et al. Impact of onset-to-reperfusion time on stroke mortality: a collaborative pooled analysis. Circulation 2013;127:1980-5.

26 Hwang YH, Kang DH, Kim YW, et al. Impact of time-to-reperfusion on outcome in patients with poor collaterals. AJNR Am J Neuroradiol 2015;36:495-500.

27 Fonarow GC, Reeves MJ, Zhao X, et al. Age-related differences in characteristics, performance measures, treatment trends, and outcomes in patients with ischemic stroke. Circulation 2010;121:879-91. 